\title{
Missed opportunities for accessing HIV care among Tshwane tuberculosis patients under different models of care
}

Running head: Antiretroviral treatment access for TB patients

G Louwagie ${ }^{1}$, B Girdler-Brown ${ }^{1}$, R Odendaal ${ }^{2}$, T Rossouw ${ }^{3}$, S Johnson ${ }^{4}$, M Van der Walt $^{2}$

\section{Affiliations}

${ }^{1}$ School of Health Systems and Public Health, University of Pretoria, Pretoria, South Africa

${ }^{2}$ TB Epidemiology and Intervention Research Unit, South African Medical Research Council, Pretoria, South Africa

${ }^{3}$ Department of Family Medicine, University of Pretoria, Pretoria, South Africa

${ }^{4}$ Foundation for Professional Development, Pretoria, South Africa

Corresponding author:

G Louwagie

Faculty of Health Sciences

School of Health Systems and Public Health

Private Bag X 323

PRETORIA, 0001 SA

South Africa

Tel: +27(0)12-354-1478

Fax: +27(0)12-354-1750

Goedele.louwagie@up.ac.za

Word count abstract : 199

Word count main body of text: 2584

No. references: 27

No. Figures: 2

No. Tables: 5 


\section{SUMMARY}

Background: This study aimed to compare access to HIV care for tuberculosis patients in settings with Antiretroviral Treatment (ART) and tuberculosis care under one roof ("semi-integrated sites") and settings with geographically separately rendered care, in Tshwane, South Africa.

Methods: Historical cohort study of patients registered with tuberculosis at 46 TB treatment points, with follow-up until the end of TB treatment. ART initiation for HIVpositive TB patients was established through linkage of TB register patient identifiers to the electronic ART register. Data analysis entailed univariate and multivariate competing risk analysis.

Results: Records of 636 and 1297 patients for semi-integrated and separate facilities respectively were reviewed. Co-trimoxazole prophylactic therapy and CD4 count recording were lower in semi-integrated than separate facilities, but the reverse was true for referral to HIV-related care.

A higher percentage of patients started ART in semi-integrated than in separate facilities $(70.5 \%$ vs. $44.6 \%, \mathrm{P}<0.001)$. In competing risk analysis (with death and LTFU as competing risks), attending a semi-integrated facility (SHR 2.49, 95\% Cl 1.06-5.88) and TB case load > 401 (SHR 1.45, 95\% Cl 1.04-2.03) were associated with increased ART initiation.

Conclusions: ART and TB treatment under one roof appears to facilitate ART initiation for HIV-positive TB patients.

Key words: tuberculosis, antiretroviral treatment, integration 
South Africa has the 3rd highest number of incident TB cases in the world. Seventy three percent of the patients with TB were estimated to be HIV-positive in $2008 .{ }^{1}$ Case-fatality in TB is substantially increased in the presence of HIV infection, even when patients receive optimal TB chemotherapy. ${ }^{2}$ Provision of Co-trimoxazole Prophylactic Therapy (CPT) and early ART initiation are critical interventions to improve outcomes in HIV-positive TB patients..$^{3-6}$ Nevertheless, only $51 \%$ of TB patients were tested for HIV in South Africa in 2009. Of the HIV-positive patients detected, $71 \%$ started or continued CPT and $42 \%$ received ART. ${ }^{7}$

One of the possible explanations for this poor performance is insufficient integration of TB and HIVIAIDS care. Proponents of integrated care argue that integrated care may increase HIV Counselling and Testing (HCT), reduce loss to follow-up and waiting time, improve staff efficiency and record keeping and reduce TB case fatality rates. $^{6,8-10}$ Integration may also increase cure rates for patients with TB if patientcentered ART adherence approaches are employed. ${ }^{11}$

Different models of HIV/TB service delivery from no integration, to several degrees of partial integration and full integration have been tried out in a variety of clinical settings in the world. ${ }^{8,11,12}$ In the South African public service context, TB and HIVIAIDS care have traditionally been rendered separately and within their own distinct cultures. ${ }^{8}$ Most primary care facilities in the public health system are nurse driven and render TB diagnostic and treatment services. They also offer TB patients HIV and CD4 count testing, CPT and referral for ART on or off-site. In the municipality where our study was undertaken, ART was initially only provided at hospitals where doctors and pharmacists are available. Later on - in an attempt to improve access to ART treatment and integrate care - ART was also introduced at some primary care facilities, albeit in a vertical fashion.

This study aimed to compare access to HIV-related care, in particular ART initiation between non-integrated or "separate facilities" (patients receiving TB and ART care at two geographically separate facilities) and "semi-integrated facilities" (TB and ART provided under the same roof, but at different consultation rooms by different health care providers) in Tshwane, the executive capital of South Africa. 


\section{MATERIALS AND METHODS}

\section{Study setting and study participants}

This was a historical cohort study. The patient population comprised of all patients diagnosed with TB and registered in the TB registers at all Tshwane public facilities from October 2008 to March 2009. All patients were followed up until the end of their TB treatment to determine ART initiation.

At the time of the study, ART initiation took place at seven hospitals and four Community Health Centres (CHCs) - all of which were supported by the Foundation for Professional Development (FPD), a private funding and teaching organization. These four CHCs were the only sites in Tshwane where both TB treatment and full ART treatment were provided at the same facility and are classified as "semiintegrated facilities" for the purpose of this study. TB patients at all other TB clinics (classified as "separate facilities") had to travel to the ART sites at the above mentioned hospitals or CHCs to start ART.

\section{Sampling size}

Sample sizes were calculated to have $80 \%$ power to detect a $10 \%$ difference in outcome proportions in semi-integrated vs. separate facilities, using single-sample z-tests, since complete sampling was used for the semi-integrated facilities. After allowing for a possible $20 \%$ missing records, the required sample size could be achieved by including all records from the semi-integrated facilities and every third record from separate facilities, over a 6-month period. TB registers were used as the sampling frame from which individual TB records were drawn.

\section{Measurements}

HIV and TB care received by patients was obtained from the standardized individual TB treatment records. Information regarding ART treatment initiation was obtained primarily from the electronic ART patient management system. This data warehouse was introduced at all public sector ART facilities in Tshwane by the FPD, with the exception of one hospital (for which the records were retrieved manually).

The ID numbers - if available - or names, place of residence and date of birth on the TB records were linked with patient identifiers on the ART registers on a one by one 
basis. Demographic, TB episode, TB case load, HCT, CD4 count at the time of TB diagnosis, referral for HIV care and CPT were obtained from the individual TB records. ART start dates were obtained from the ART database.

Eligibility for ART treatment was defined as having a CD4 count of 200 cells $/ \mu$ or less and did not include WHO staging. Although the guidelines enforced at the time of the study also made provision for ART initiation in Stage IV disease,$^{13}$ our study assessed the practice of nurses - who were not trained to do clinical staging of ART patients and did not capture staging on TB records. Data collection and linkage were piloted at two clinics.

\section{Data analysis and management}

Data were single entered in Epi-Info by full-time data capturers under the supervision of an experienced data manager. Check files built into Epi Info and a programme written in Stata version 11 were used to reduce capturing errors. ${ }^{14}$ Data analysis was performed using Stata version $11 .{ }^{14}$ Descriptive summary statistics were calculated as means with their standard deviations, as proportions, and as medians with inter-quartile ranges, as appropriate. Comparisons of unadjusted proportions were carried out using a single sample two-sided z-test.

The study subjects for the competing risk survival analysis were all those who met the criteria for ART eligibility but excluding those who transferred in or out. Our outcome of interest was having started ART, as recorded in the ART data base. TB treatment outcomes "death" or "defaulted" were classed as a "competing risk". Patients who did not start ART, or neither died nor defaulted were censored at the recorded TB treatment outcome date.

The following explanatory co-variables were considered for inclusion in the competing risks regression model: semi-integrated vs. separate facilities; yearly number of TB cases seen in the clinic; type of health care facility; new or retreatment TB case; sex; age; CD4 count; type of TB and whether the patient received directly observed treatment or not. Co-variables with P-values of less than 0.25 were then retained - following recommendations for multiple binary logistic regression - for inclusion in the competing risks regression model. ${ }^{15}$ Interaction terms were generated between the semi-integrated vs. separate variable and each of the other retained co-variables and eliminated using backwards hierarchical 
regression. ${ }^{16}$ Results were adjusted for clustering on TB facilities using robust standard errors.

\section{Ethical approval}

The study was approved by the Ethics committees of the University of Pretoria and the South African Medical Research Council.

\section{RESULTS}

TB data were collected for 46 TB treatment points. Eighty two percent of TB patient records could be traced for the time period of the study: of these 636 records for the four semi-integrated sites and 1298 for the 42 separate sites were sampled. The record of one outlier with an implausible long TB treatment duration was excluded from the analysis. Only 933 (48.2\%) of the TB records had an ID number, but most ART records did. TB records were linked on a one to one basis to over close to 73000 records on the ART database.

\section{Baseline characteristics of registered TB patients by facility type}

The median age of patients was 35 for semi-integrated and 34 years for separate facilities and nearly half were female. Over $93.3 \%$ of the patients presented with their first episode of TB. A higher percentage of patients presented with Pulmonary Tuberculosis at semi-integrated facilities than at the separate facilities $(86.2 \%$ vs. $80.6 \%$ ). DOT supervision in the intensive phase was higher in separate facilities than in semi-integrated facilities ( $86.7 \%$ vs. $72.3 \%$ ). Median CD4 counts were very similar in both types of facilities, a finding to be interpreted with some caution since values were available for less than half of the HIV-positive patients (Table 1).

\section{HIV counselling, testing and non-ART HIV-related care by facility type}

At both semi-integrated and separate facilities a high percentage of patients knew that they were HIV sero-positive by the time they registered for TB treatment $(39.8 \%$ and $32.8 \%$ ). For the remainder, $78.6 \%$ and $81.8 \%$ were counselled at semiintegrated and separate facilities respectively. Most counselled patients were subsequently tested at both facility types (90.7\% and $89.1 \%$ ). Overall $70.3 \%$ of 
Table 1 Tuberculosis records: characteristics of study subjects*

\begin{tabular}{lrrrr} 
& \multicolumn{2}{c}{$\begin{array}{c}\text { Semi-integrated facility } \\
(\mathrm{N}=636: \text { complete sampling })\end{array}$} & \multicolumn{2}{c}{$\begin{array}{c}\text { ART at separate facility } \\
(\mathrm{N}=1297: \text { sampling 1 in 3) }\end{array}$} \\
\hline Age (Median [IQR]) & $\mathrm{N}=632$ & $35(27-44)$ & $\mathrm{N}=1288$ & $34(26-43)$ \\
Female & $310 / 629$ & $49.3 \%$ & $618 / 1281$ & $48.2 \%$ \\
First episode of TB & $598 / 629$ & $95.1 \%$ & $1203 / 1290$ & $93.3 \%$ \\
PTB (including PTB + EPTB) & $531 / 616$ & $86.2 \%$ & $990 / 1229$ & $80.6 \%$ \\
Newly registered ${ }^{\dagger}$ & $593 / 634$ & $93.5 \%$ & $1125 / 1266$ & $88.9 \%$ \\
DOT intensive phase & $460 / 636$ & $72.3 \%$ & $1124 / 1297$ & $86.7 \%$ \\
HIV sero-status Known +ve & $253 / 636$ & $39.8 \%$ & $425 / 1297$ & $32.8 \%$ \\
$\quad$ New +ve & $194 / 636$ & $30.5 \%$ & $411 / 1297$ & $31.7 \%$ \\
$\quad$ Negative & $74 / 636$ & $11.6 \%$ & $212 / 1297$ & $16.4 \%$ \\
$\quad$ Unknown & $115 / 636$ & $18.1 \%$ & $249 / 1297$ & $19.2 \%$ \\
CD4 count (Median [IQR]) & & & & $120.5(51-226.3)$ \\
$\quad$ All HIV +ve & $\mathrm{N}=171$ & $123(57-195)$ & $\mathrm{N}=450$ & $108(52.3-203.5)$ \\
\hline
\end{tabular}

${ }^{*}$ All information obtained from TB registers and individual TB patient records

ti.e. not transferred in or moved in

$\mathrm{HIV}=$ human immunodeficiency virus; $\mathrm{TB}=$ tuberculosis; $\mathrm{N}=$ sample size; $\mathrm{SD}=$ standard deviation; $\mathrm{PTB}=$ pulmonary tuberculosis; EPTB = extra-pulmonary tuberculosis; DOT = directly observed treatment; +ve = positive; CD4 = CD4 count in cells/ $\mu$ l of blood; IQR = inter-quartile range.

Table 2 HIV counselling, testing and care by type of facility*

\begin{tabular}{|c|c|c|c|c|c|}
\hline \multirow{2}{*}{$\begin{array}{l}\text { Variable } \\
\text { Counselled if not known +ve }\end{array}$} & \multicolumn{2}{|c|}{$\begin{array}{l}\text { Semi-integrated facility } \\
\qquad(\mathrm{N}=636)\end{array}$} & \multicolumn{2}{|c|}{$\begin{array}{l}\text { ART at separate facility } \\
\qquad(\mathrm{N}=1297)\end{array}$} & \multirow{2}{*}{$\begin{array}{c}\mathrm{P}^{\dagger} \\
0.015\end{array}$} \\
\hline & $301 / 383$ & $78.6 \%$ & $713 / 872$ & $81.8 \%$ & \\
\hline Tested if counselled ${ }^{\ddagger}$ & $273 / 301$ & $90.7 \%$ & $635 / 713$ & $89.1 \%$ & 0.172 \\
\hline CPT if HIV +ve & $289 / 447$ & $64.7 \%$ & $573 / 836$ & $68.5 \%$ & 0.018 \\
\hline CCMT referral & $194 / 447$ & $43.4 \%$ & $132 / 836$ & $15.8 \%$ & $<0.001$ \\
\hline \multicolumn{6}{|l|}{ Recorded CD4 count } \\
\hline Known +ve not on prior ART & $43 / 215$ & $20.0 \%$ & $63 / 363$ & $16.8 \%$ & 0.104 \\
\hline Known +ve on prior ART & $9 / 38$ & $23.7 \%$ & $23 / 62$ & $37.1 \%$ & 0.033 \\
\hline New HIV +ve & $85 / 194$ & $43.8 \%$ & $244 / 411$ & $59.4 \%$ & $<0.001$ \\
\hline
\end{tabular}

${ }^{*}$ All information obtained from TB registers and individual TB patient records

${ }^{\dagger} \mathrm{P}$-values for single sample, 2-tail z-tests

${ }^{\ddagger}$ No patients were tested without counselling at either facility type

$\mathrm{HIV}=$ human immunodeficiency virus; $\mathrm{N}=$ sample size; +ve = positive; CCMT = HIV comprehensive care,

management and treatment programme; CD4 = CD4 cell count/ $\mu \mathrm{l}$ of blood. 
patients were HIV-positive, $11.6 \%$ HIV-negative and $18.1 \%$ had an unknown status for the semi-integrated facilities. At separate facilities $64.5 \%$ of patients were HIVpositive, $16.3 \%$ HIV-negative and $19.2 \%$ had an unknown HIV status. Separate facilities performed considerably better in terms of CPT and more often recorded CD4 counts for newly diagnosed HIV positive patients and for patients on prior ART. Conversely, semi-integrated facilities much more often recorded referring patients for HIV care (Figure 1, Table 1 and Table 2).

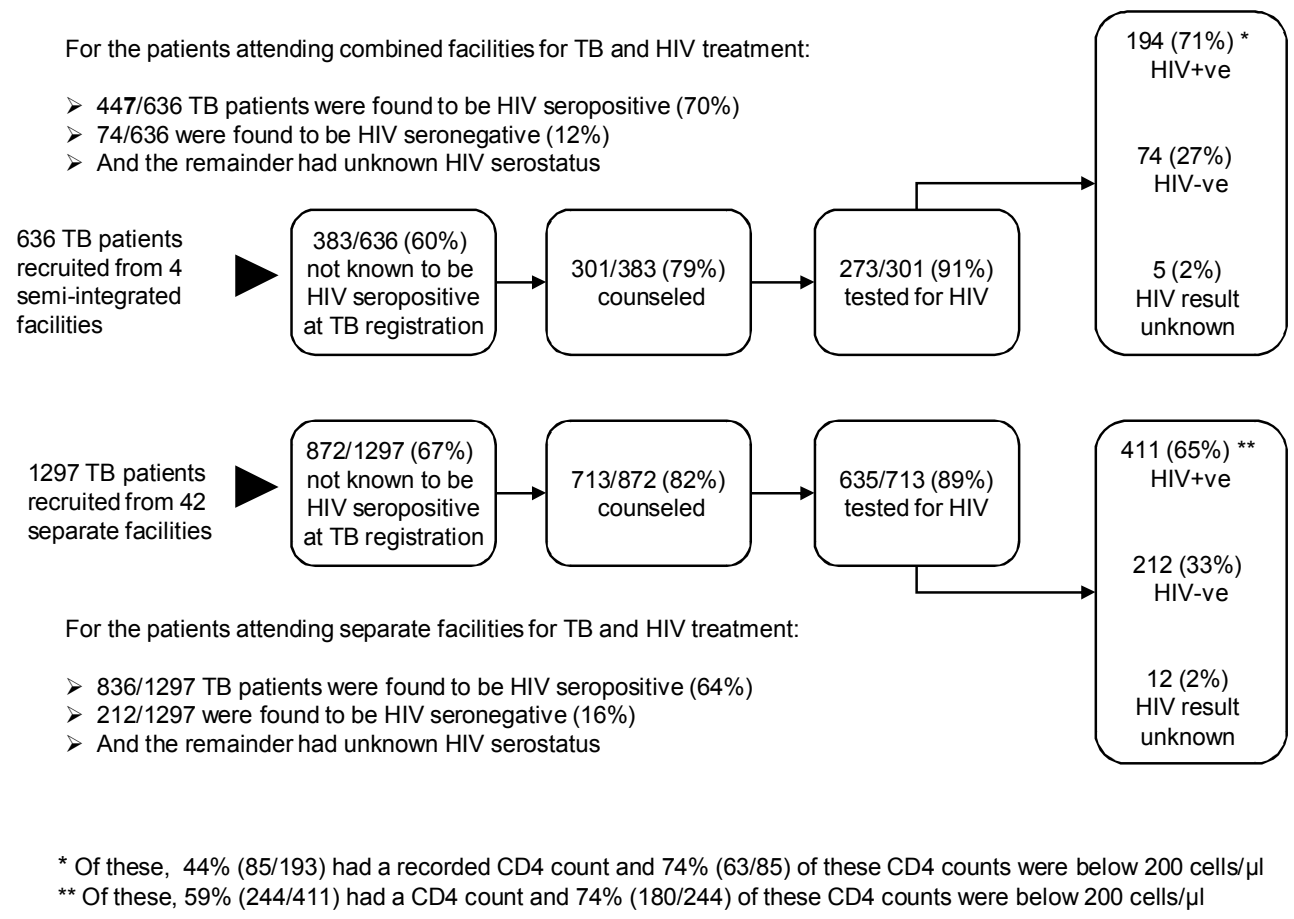

Figure 1 HIV counselling, testing and HIV-positivity rates at semi-integrated vs. separate facilities

\section{ART initiation by facility type}

Table 3 presents the unadjusted results for ART initiation for both types of facilities. After excluding TB patients who were already on ART prior to being diagnosed with TB, patients with a CD4 count $\geq 200$ cells/ $\mu$ l or a missing CD4 count and patients transferred in or out, 105 and 233 patients were available for analysis in the semi- 
Table 3 ART initiation by type of facility for HIV-positive patients

\begin{tabular}{|c|c|c|c|c|c|}
\hline \multirow{2}{*}{$\begin{array}{l}\text { Variable } \\
\text { Not on ART prior to TB treatment }\end{array}$} & \multicolumn{2}{|c|}{$\begin{array}{l}\text { Semi-integrated facility } \\
\qquad(N=447)\end{array}$} & \multicolumn{2}{|c|}{$\begin{array}{l}\text { ART at separate facility } \\
\qquad(\mathrm{N}=836)\end{array}$} & \multirow[t]{2}{*}{$P^{*}$} \\
\hline & $409 / 447$ & $91.5 \%$ & $774 / 836$ & $92.6 \%$ & \\
\hline No CD4 count recorded & $281 / 409$ & $68.7 \%$ & $469 / 774$ & $60.6 \%$ & \\
\hline With CD4 count $<200$ & $117 / 409$ & $28.6 \%$ & $284 / 774$ & $36.7 \%$ & \\
\hline Not transferred/moved in/out & $105 / 117$ & $89.7 \%$ & $233 / 284$ & $82.0 \%$ & \\
\hline$\%$ of these patients starting $\mathrm{ART}^{\dagger}$ & $74 / 105$ & $70.5 \%$ & $104 / 233$ & $44.6 \%$ & $<0.001^{* \dagger}$ \\
\hline $\begin{array}{l}\text { Time to ART initiation (Median } \\
{[I Q R] \text { ) }}\end{array}$ & \multicolumn{2}{|c|}{$67.5(53-92)$} & \multicolumn{2}{|r|}{$67(35-109.5)$} & $0.995^{\star *}$ \\
\hline
\end{tabular}

*P-value for single sample 2-tail z-test, **Wilcoxon ranksum test

${ }^{\dagger}$ Crude (unadjusted) Risk Ratio = 1.58 (95\% Confidence interval: 1.31-1.91: chi square $\mathrm{P}<0.001$ )

$\mathrm{ART}=$ antiretroviral treatment; $\mathrm{HIV}=$ human immunodeficiency virus; $\mathrm{TB}=$ tuberculosis; $\mathrm{CD} 4=\mathrm{CD} 4 \mathrm{cells} / \mu \mathrm{l}$ blood.

integrated and separate facilities respectively. Although there was no difference in median time to ART initiation, a significantly higher percentage initiated ART at semi-integrated facilities than at separate facilities $(70.5 \%$ vs. $44.6 \%, \mathrm{P}<0.001)$. The difference in the cumulative proportion of patients initiating ART at different time points is presented in Figure 2.

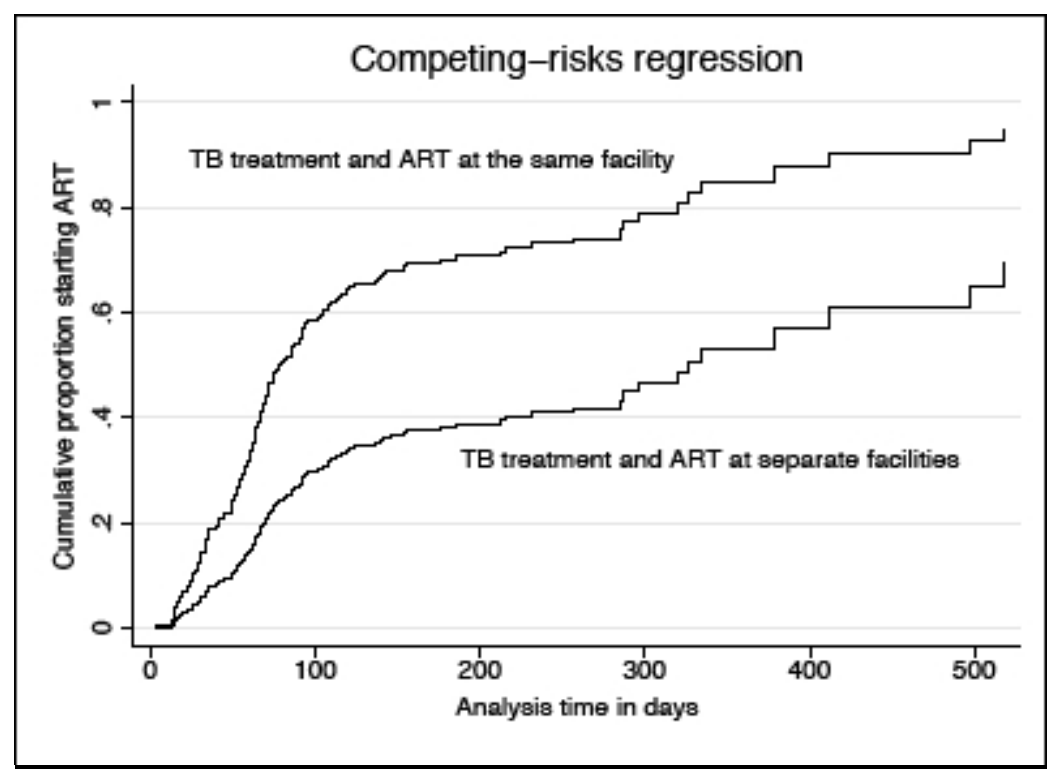

Figure 2 Cumulative proportion of patients starting on ART in semi-integrated vs. separate facilities

Note: $P$-value for subhazard ratio comparing semi-integrated with separate facilities $=0.0006$ 
Table 4 Tuberculosis records: subjects considered for competing risks regression*

\begin{tabular}{|c|c|c|c|c|}
\hline \multirow[b]{2}{*}{ Age (Mean [SD]) } & \multicolumn{2}{|c|}{$\begin{array}{l}\text { Semi-integrated facility } \\
\qquad(\mathrm{N}=105)\end{array}$} & \multicolumn{2}{|c|}{$\begin{array}{l}\text { ART at separate facility } \\
\qquad(\mathrm{N}=233)\end{array}$} \\
\hline & $N=104$ & $37.3(8.5)$ & $\mathrm{N}=232$ & $36.8(10.2)$ \\
\hline Female & $63 / 105$ & $60.0 \%$ & $106 / 229$ & $46.3 \%$ \\
\hline First episode of TB & $102 / 105$ & $97.1 \%$ & $218 / 233$ & $93.6 \%$ \\
\hline PTB (incl. PTB + EPTB) & $85 / 101$ & $84.2 \%$ & $185 / 226$ & $81.9 \%$ \\
\hline DOT intensive phase & $85 / 105$ & $81.0 \%$ & $199 / 233$ & $85.4 \%$ \\
\hline Newly diagnosed HIV +ve & $53 / 105$ & $50.5 \%$ & $145 / 233$ & $62.2 \%$ \\
\hline CD4(Median [IQR]) All HIV +ve & $\mathrm{N}=105$ & $78(44.5-135.5)$ & $\mathrm{N}=233$ & $77(36.5-123)$ \\
\hline New HIV +ve & $N=53$ & $74(46-118)$ & $\mathrm{N}=145$ & $78(44-124)$ \\
\hline
\end{tabular}

*All information obtained from TB registers and individual patient records: all subjects neither transferred/moved in/out and with CD4 counts $<200$ cells/ $\mu$ l

$\mathrm{HIV}=$ human immunodeficiency virus; $\mathrm{TB}=$ tuberculosis; $\mathrm{N}=$ sample size; $\mathrm{SD}=$ standard deviation; $\mathrm{PTB}=$ pulmonary tuberculosis; EPTB = extra-pulmonary tuberculosis; DOT = directly observed treatment; +ve = positive; CD4 = CD4 count in cells/ $\mu$ l of blood; IQR = inter-quartile range.

Table 5 Competing risks regression results*

\begin{tabular}{|c|c|c|c|c|c|c|}
\hline & \multicolumn{2}{|c|}{ Univariate analysis } & \multirow{2}{*}{\multicolumn{4}{|c|}{$\begin{array}{l}\text { Multivariate analysis } \\
\text { 95\% RCI for ASHR }\end{array}$}} \\
\hline & \multirow[b]{2}{*}{ CSHR } & \multirow[b]{2}{*}{$P>z$} & & & & \\
\hline & & & ASHR & Lower & Upper & $P>z$ \\
\hline Semi-integrated vs. separate facility ${ }^{\ddagger}$ & 1.97 & 0.006 & 2.49 & 1.06 & 5.88 & 0.037 \\
\hline >401 vs. <402 TB patients/year & 1.85 & 0.039 & 1.45 & 1.04 & 2.03 & 0.029 \\
\hline TB treating facility type $\mathrm{e}^{\S}$ & & & & & & \\
\hline Provincial community health & & & & & & \\
\hline centre & Reference & & Reference & & & \\
\hline Municipal clinic & 0.54 & 0.019 & 1.37 & 0.57 & 3.27 & 0.476 \\
\hline Provincial clinic & 0.67 & 0.148 & 1.79 & 0.72 & 4.41 & 0.209 \\
\hline
\end{tabular}

* 338 Subjects were neither transferred in or out and were eligible to start on ART; 336 of these study subjects had full data available for multivariate competing risks regression. ${ }^{\$}$ Only variables with a P-value $<0.25$ in the univariate analysis are presented here. ${ }^{\S}$ Some facilities are managed by provincial authorities and others by the municipality.

$\mathrm{RCI}=$ robust confidence interval; $\mathrm{ASHR}$ = adjusted subhazard ratio; CSHR = crude subhazard ratio; $\mathrm{TB}=$ tuberculosis

The baseline characteristics of the patients considered for inclusion in the competing risk analysis are compared in Table 4. Age distribution, type of TB and median CD4 counts were similar, but there appeared to be some differences for percentage of females, DOT in the intensive phase and having a first episode of TB vs. retreatment TB. In multivariate competing risk regression analysis patients attending semi-integrated services were significantly more likely to initiate ART than 
patients who had to travel to another facility to receive ART (Sub Hazard Ratio [SHR] 2.49, 95\%Cl 1.06-5.88). Patients attending facilities with a TB caseload of over 401 new patients per year ( 3 separate and 2 semi-integrated facilities fell in this category) were also more likely to initiate ART (SHR 1.45, 95\% Cl 1.04-2.03) (Table $5)$.

\section{DISCUSSION}

Eligible HIV-positive TB patients attending semi-integrated facilities were more likely to initiate ART than patients who had to travel to another facility to receive ART (SHR 2.49, $\mathrm{P}=0.037$ ). This confirms our hypothesis that providing TB and ART care at different facilities poses barriers to patients that may prevent them from accessing life saving treatment. Reduced access to HIV care for TB patients in a nonintegrated system was also demonstrated in two studies that compared off- and onsite voluntary counselling and testing in the Democratic Republic of Congo and Cambodia. ${ }^{17,18}$ Okot-Chono et al. found that only $66 \%$ of 333 adult TB patients were tested for HIV in Uganda, and that only $12 \%$ of the HIV-positive patients were on both ART and CPT and about a third had not received any HIV care, mostly due to limited TB-HIV inter-clinic referral and poor service integration. ${ }^{19}$ Similar findings came out of India where only between $30 \%$ and $38 \%$ of HIV-infected TB patients started ART during treatment. ${ }^{20,21}$ Our findings support the call of many researchers to integrate HIV and TB services. ${ }^{9,22,23}$ The beneficial effect of integrating ART and TB services in one facility (whether fully or partially) has been demonstrated in a variety of settings. ${ }^{11,24-26}$

In this study, a high percentage of patients knew their HIV status by the time they registered for TB treatment at both types of facilities and HIV counselling and testing rates were high. CPT was somewhat less well recorded and may point towards either poor recording or poor provision of this essential prophylactic medication. Separate facilities recorded CPT and CD4 counts more often than semi-integrated facilities. This may be explained by the fact that information about CPT and CD4 results is kept in ART files and not in the TB files at semi-integrated facilities, for those patients who have enrolled for ART. This calls for having only one file per patient, a system already successfully introduced at ART sites in Khayelitsha, South 
Africa. ${ }^{11}$ Referral for HIV care was recorded more often at semi-integrated facilities: this appears logical since referral is easier if ART is provided at the same facility. In terms of other predictors of ART initiation, patients attending facilities with a larger TB caseload were more likely to initiate ART independent of whether the facility had ART on site or not. The reasons for this are not clear but it may be that professional nurses who see more TB patients are also more experienced in HIV-related care and the need for referral for ART initiation.

Our study has several limitations. Missing records and patients linked to the wrong patients in the ART database (due to spelling and recording errors) may have introduced bias. This is a retrospective record review: it is therefore difficult to distinguish between a service "not rendered" and a service "rendered, but not recorded". Some underestimation of ART initiation is possible. Our figures regarding ART initiation are solely based on the ART data collected for the Tshwane public sector facilities. Some patients may have received ART outside Tshwane, at the workplace or from private practitioners. There is however no reason to believe that patients' health seeking behaviour outside the public sector would differ for patients attending the two types of facilities. Our finding of differential ART initiation is therefore likely to remain valid. Conversely, we may have over-estimated ART initiation. Some of the patients who did not have a CD4 count test result recorded on their TB records may have been eligible for ART. A sensitivity analysis was carried out involving all HIV-positive TB patients, irrespective of CD4 count results, who were not on prior ART, and were not transferred in or out. Among these subjects, $47.5 \%(158 / 333)$ started on ART before the end of their TB treatment at semiintegrated facilities vs. $30.4 \%(186 / 612)$ at separate facilities $(P<0.0001)$ with a crude risk ratio of $1.56(95 \% \mathrm{Cl} 1.32$ to 1.84$)$. It is important to note that under these assumptions, ART initiation is low even at semi-integrated facilities. This may mean that vertical organization of TB and HIV care creates hurdles for patients to access care even within the same facility. Furthermore, our study results may not be generalisable to settings without external funding support.

\section{CONCLUSION AND RECOMMENDATIONS}

To our knowledge, this study was the first of its kind to compare semi-integrated with non-integrated facilities for ART initiation. The electronic data made it possible 
to link TB and HIV information from the TB sites to all public sector ART sites, something which would have been extremely cumbersome with manual ART records.

HIV counselling and testing of TB patients is well established in both types of facilities. However, recording and/or provision of CPT and CD4 testing needs improvement, particularly in semi-integrated sites. ART initiation for eligible HIVpositive TB patients was higher in semi-integrated facilities than in separate facilities. This finding supports the call for wider provision of ART care at primary care facilities.

We recommend the introduction of a "one patient, one file, one appointment" principle at all facilities that provide TB and ART services. This will facilitate a shift away from functions and tasks to 'patient-centered care', ${ }^{27}$ integrated monitoring systems and earlier ART initiation in HIV-positive TB patients with subsequent reduced mortality. ${ }^{4-6}$

\section{ACKNOWLEDGEMENTS}

This project was supported by funding from the South African National Department of Health (Grant 231/209-2010) and by the South African Medical Research Council. All co-authors contributed to the conception and design of the study and critically revised the article for intellectual content. BGB performed statistical analysis, RO was responsible for data acquisition and management, SJ for ART warehouse support and MVDW for the overall operational management of the project.

The authors wish to thank Professor Piet Becker of the Medical Research Council for his statistical advice and the Tshwane HIV and TB managers and TB nurses for supporting this project. We also acknowledge the Foundation for Professional Development for giving us access to the data in the ART warehouse, in particular Mr. Veli Mnisi, manager of the SOZO data warehouse. 


\section{REFERENCES}

1 World Health Organization. Global Tuberculosis Control: WHO report 2010. Report No: WHO/HTM/TB/2010.7. Geneva: World Health Organization, 2009.

2 Mukadi YD, Maher D, Harries A. Tuberculosis case fatality rates in high HIV prevalence populations in Sub-Saharan Africa. AIDS 2001; 15: 143-152.

3 Harries AD, Zachariah R, Corbett EL, et al. The HIV-associated tuberculosis epidemic-when will we act? Lancet 2010; 375: 1906-1919.

4 Abdool Karim SS, Naidoo K, Grobler A, et al. Timing of initiation of antiretroviral drugs during tuberculosis therapy. N Engl J Med 2010; 362: 697-706.

5 Tabarsi P, Saber-Tehrani AS, Baghaei $P$, et al. Early initiation of antiretroviral therapy results in decreased morbidity and mortality among patients with TB and HIV. J Int AIDS Soc 2009; 12: 14.

6 Blanc F, Sok T, Laureillard D, et al. Early (2 weeks) vs. late (8 weeks) initiation of highly active antiretroviral treatment (HAART) significantly enhances survival of severely immunosuppressed HIV-infected adults with newly diagnosed tuberculosis: results of the CAMELIA clinical trial. BMC Proceedings 2011; 5 (Suppl 1): 011.

7 World Health Organization. Global Tuberculosis Control. A Short Update to the 2009 Report. Report No. WHO/HTM/TB/2009.426. Geneva: World health Organization, 2010.

8 Coetzee D, Hilderbrand K, Goemaere E, Matthys F, Boelaert M. Integrating tuberculosis and HIV care in the primary care setting in South Africa. Trop Med Int Health 2004; 9: A11-A15.

9 Abdool-Karim SS, Abdool-Karim Q, Friedland G, Lalloo U, El-Sadr WM; START project. Implementing antiretroviral therapy in resource-constrained settings: opportunities and challenges in integrating HIV and tuberculosis care. AIDS 2004; 18: 975-979.

10 Perumal R, Padayatchi N, Stiefvater E. The whole is greater than the sum of the parts: Recognising missed opportunities for an optimal response to the rapidly maturing TB-HIV co-epidemic in South Africa. BMC Public Health; 2009; 9: 243. 
11 Friedland G, Harries A, Coetzee D. Implementation issues in tuberculosis/HIV program collaboration and integration: 3 case studies. J Infect Dis 2007; 196(Suppl 1): S114-S123.

12 Harris JB, Hatwiinda SM, Randels KM, et al. Early lessons from the integration of tuberculosis and HIV services in primary care centers in Lusaka, Zambia. Int J Tuberc Lung Dis 2008; 12: 773-779.

13 National Department of Health, RSA. National Antiretroviral Treatment Guidelines, $1^{\text {st }}$ edition, 2004. Pretoria: DOH, 2010.

<http://www.doh.gov.za/docs/factsheets/guidelines/artguide04-f.html > (Last Accessed 1602 2011).

14 Stata Corp. Stata Statistical Software: Release 11. College Station, Texas: StataCorp LP, 2009.

15 Hosmer DW, Lemeshow S. Applied Logistic Regression. Second edition. New York: John Wiley and Sons, 2000.

16 Kleinbaum DG, Klein M. Logistic Regression: A Self-Learning Text. Second edition. New York: Springer, 2002.

17 Van Rie A, Sabue M, Jarrett N, et al. Counselling and testing TB patients for HIV: Evaluation of three implementation models in Kinshasa, Congo. Int J Tuberc Lung Dis 2008; 12 (Suppl 1): 73-78.

18 Kanara N, Cain KP, Chhum V, et al. Association between distance to HIV testing site and uptake of HIV testing for tuberculosis patients in Cambodia. Int J Tuberc Lung Dis 2009; 13: 226-231.

19 Okot-Chono R, Mugisha F, Adatu F, Madraa E, Dlodlo R, Fujiwara P. Health system barriers affecting the implementation of collaborative TB-HIV services in Uganda. Int J Tuberc Lung Dis 2009; 13: 955-961.

20 Thomas BE, Dewan P K, Vijay S, et al. Perceptions of tuberculosis patients on provider-initiated HIV testing and counselling-a study from South India. PLoS One 2009; 4: e8389.

21 Raizada N, Chauhan LS, Babu BS, et al. Linking HIV-infected TB patients to cotrimoxazole prophylaxis and antiretroviral treatment in India. PLoS One 2009; 4: e5999.

22 Zachariah R, Teck R, Ascurra O, et al. Can we get more HIV-positive tuberculosis patients on antiretroviral treatment in a rural district of Malawi? Int J Tuberc Lung Dis 2005; 9: 238-247. 
23 Wood R. The case for integrating tuberculosis and HIV treatment services in South Africa. J Infect Dis 2007; 196(Suppl 3): S497-S499.

24 Huerga $H$, Spillane $H$, Guerrero W, Odongo A, Varaine F. Impact of introducing human immunodeficiency virus testing, treatment and care in a tuberculosis clinic in rural Kenya. Int J Tuberc Lung Dis 2010; 14: 611-615.

25 Friedland G, Abdool Karim S, Abdool Karim Q, et al. Utility of tuberculosis directly observed therapy programs as sites for access to and provision of antiretroviral therapy in resource-limited countries. Clin Infec Dis 2004; 38(Suppl 5): S421-S428.

26 Jack C, Lalloo U, Karim QA, et al. A pilot study of once-daily antiretroviral therapy integrated with tuberculosis directly observed therapy in a resourcelimited setting. JAIDS 2004; 36: 929-934.

27 Church K, Lewin S. Delivering integrated HIV services: Time for a clientcentred approach to meet the sexual and reproductive health needs of people living with HIV? AIDS 2010; 24:189-193. 